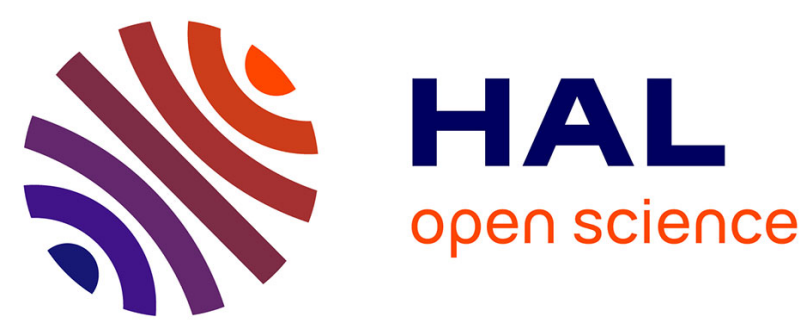

\title{
On the estimation of the consensus rate of convergence in graphs with persistent interconnections
}

Nilanjan Roy Chowdhury, Srikant Sukumar, Mohamed Maghenem, Antonio

Loria

\section{- To cite this version:}

Nilanjan Roy Chowdhury, Srikant Sukumar, Mohamed Maghenem, Antonio Loria. On the estimation of the consensus rate of convergence in graphs with persistent interconnections. International Journal of Control, 2018, 91 (1), pp.132-144. 10.1080/00207179.2016.1272006 . hal-01744659

\author{
HAL Id: hal-01744659 \\ https://hal.science/hal-01744659
}

Submitted on 5 Mar 2020

HAL is a multi-disciplinary open access archive for the deposit and dissemination of scientific research documents, whether they are published or not. The documents may come from teaching and research institutions in France or abroad, or from public or private research centers.
L'archive ouverte pluridisciplinaire HAL, est destinée au dépôt et à la diffusion de documents scientifiques de niveau recherche, publiés ou non, émanant des établissements d'enseignement et de recherche français ou étrangers, des laboratoires publics ou privés. 


\title{
On the estimation of the consensus rate of convergence in graphs with persistent interconnections
}

\author{
Nilanjan Roy Chowdhury*, Srikant Sukumar*, Mohamed Maghenem ${ }^{\dagger}$ Antonio \\ Loría $^{\dagger}$
}

\begin{abstract}
The aim of the current article is to establish myriad convergence rate estimates to consensus for timevarying graphs with persistent interaction. Several novel analysis methodologies for consensus protocols employing the notions of persistence of excitation and Lyapunov functions are provided.The estimates are compared with each other and existing literature [7]. Numerical simulations on test examples are illustrated to support the theoretical findings.
\end{abstract}

\section{Index Terms}

Consensus, Time-varying systems, Gradient descent, Persistence of excitation, Exponential stability.

\section{INTRODUCTION}

Estimating the speed at which consensus is reached in a network of dynamical systems, has drawn significant attention in the past few years. For graphs with time-invariant interconnections it is determined by the smallest non-zero eigen-value of the Laplacian matrix, referred to as algebraic connectivity. See, e.g., [9] where an estimate of the convergence rate to consensus

* N. Roy Chowdhury (Research scholar) and S. Srikant (Assistant professor) are with the Department of Systems and Control Engineering, Indian Institute of Technology Bombay, Mumbai-400076, India. Email: nilanjan@sc.iitb.ac.in, srikant@sc.iitb.ac.in

$\dagger$ M. Maghenem is with Univ. Paris Sud. A. Loría is with CNRS. Address: LSS-SUPELEC, 91192 Gif-sur-Yvette, France. E-mail: mohamed.maghenem@1ss.supelec.fr, antonio.loria@1ss.supelec.fr 
is established for a class of double-integrator autonomous heterogeneous systems using linear systems theory.

For graphs with time-varying topologies and/or interconnections, estimating the rate of convergence is a much harder task since the graph dynamics is linear time-varying hence, eigen-value analysis is insufficient. In [4], for the discrete-time agreement protocol, under the assumption that the time-varying graph is B-connected (the union of $B$ non-simultaneous "snap shots" of the graph is connected) and the Laplacian matrix is stochastic at any instant, the rate of convergence is computed using the joint spectral radius of the Laplacian matrix taken at succesive instances. An (increasing) estimate of the convergence time in terms of the number of nodes and $B$ is given in [16]. In [6] the notion of stochastic indecomposable aperiodic matrices is used to estimate the consensus rate of convergence for discrete-time switching graphs.

For time-varying graphs expressed in continuous time, perhaps the most general result remains that of the seminal paper [14]. In the latter, consensus is established under the condition that there exists at least one spanning tree with a persistently-exciting Laplacian. The proof, however, is rather involved as it uses non-differentiable min-max Lyapunov functions and the rate of convergence is not established. In [10] consensus is established under the so-called cut-balance interaction assumption. This means that the ratio of the sums of reciprocal interconnection weights (that is the weights of interconnections from and to each agent) is bounded. In [12] this assumption was relaxed to allow for reciprocal interaction weights to be indefinitely far apart that is, the ratio slowly diverges to infinity. An explicit convergence bound is given for the maximal difference between any pair of agents' states.

In [17] is proposed a continuous and a discrete time update law to achieve consensus for singleintegrator agent dynamics communicating via directed persistent graphs that is, graphs for which the functions that quantify the strength of each interconnection is non-integrable. Furthermore, under the so-called arc-balance assumption a convergence rate is estimated in function of the cycle edges i.e., arcs that are not persistent. More recently, inspired by theory of stability for linear time-varying systems, a convergence rate estimate was provided in [7] for graphs with persistent connectivity (it is assumed that the weight-functions are persistently exciting [15]).

In this paper, we present several convergence-rate estimates for networks of systems interconnected through persistently-excited graphs. That is, we assume that the agents interact through time-varying interconnections which, individually, are persistently exciting. Roughly speaking, 
the graph is not guaranteed to be connected for any instant $t$ but only "in average" over a sliding finite window of time of fixed length. Our results, which apply to both, networks of single and double-integrator dynamics, rely on stability theory of so-called gradient-descent systems, known in adaptive control theory. That is, we show how the classical consensus paradigm may be recasted in a problem of stability analysis for systems with persistency of excitation.

Our analysis relies on the edge-agreement transformation proposed in [7], based on the relationship between node and edge-agreement protocol [13]. Loosely speaking, this transformation allows to "extract" the information of an arbitrary underlying spanning tree which defines a dynamic system whose stability at the origin implies consensus. Four different convergence estimates are established; three of them follow lines of proof of statements for linear timevarying systems [5], [1], [11]. The fourth, self-contained, is based on the construction of a strict Lyapunov function for the gradient system. A preliminary version of some of these results was presented in [8].

The rest of the paper is organized as follows. In the following section we introduce some notations and show how the consensus problems for single and double-integrator dynamics may be recasted in the stability analysis of a unique differential equation: the gradient system. Our main statements, giving the explicit convergence rates, are presented in Section III. Numerical simulations that illustrate our theoretical findings are provided in Section IV and the paper is wrapped up with some concluding remarks in Section V.

\section{NETWORK MODEL}

\section{A. Preliminaries}

Following the notation of [13] a graph $\mathcal{G}$ consists a set of vertices and edges; the former are denoted by $v_{i}$ with $i \leq n$ and, for each $j \leq m \leq n(n-1)$ (assuming no self-edges) the edge $e_{j}:=\left\{v_{k}, v_{l}\right\}$ links the node $v_{k}$ to $v_{l}$ for some $k, l \leq n$ (the vertices and edges being labeled arbitrarily, the indexes $k$ and $l$ do not necessarily coincide with $j$ ). The edge is said to be directed if the sense in which the information flows is important; in which case, the edge links a tail node to a head node.

A sequence of adjacent vertices $\left\{v_{i}, v_{i+1}, \cdots, v_{i+l}\right\}$ with $l \leq n-i$ forms a path of length $l$. A path forms a simple cycle if the path is closed that is, if $v_{i}=v_{i+l}$. A graph is connected, if for every pair of vertices there is a path that has them as its end vertices. A tree is defined as a 
connected graph without cycles; it is a spanning tree if it contains all the vertices in the graph. In this article we use $p:=n-1$ to denote the number of spanning tree edges.

Without distinction of the flow of information, the graph is said to be undirected. Now, by arbitrarily assigning an orientation to an otherwise undirected graph $\mathcal{G}$, one may compute the incidence matrix $D(\mathcal{G}) \in \mathbb{R}^{n \times m}$ which characterizes the graph's structure. The elements of $D$, denoted by $d_{i j}$, are defined as follows. For each edge $e_{j}, d_{i j}=-1$ if $v_{i}$ is the tail of $e_{j}, d_{i j}=1$ if $v_{i}$ is the head of $e_{j}$, and $d_{i j}=0$ if $v_{i} \notin e_{j}$.

In addition to the incidence matrix, the strength of each interconnection is characterized by an edge weight, denoted by $w_{i}(\cdot)$ with $i \leq m$. The edge weights may be constant or functions of time and the state. These coeeficients are collected in the diagonal matrix $W:=\operatorname{diag}\left[w_{i}(\cdot)\right] \in \mathbb{R}^{m \times m}$.

Thus, the graph's Laplacian matrix $L \in \mathbb{R}^{n \times n}$ may be defined as a function of the weights matrix and the incidence matrix as $L(\mathcal{G})=D(\mathcal{G}) W D(\mathcal{G})^{\top}$. For any arbitrarily oriented undirected graph the latter equation computes to the same Laplacian matrix. Likewise, the edge Laplacian $\tilde{L}_{e}(\mathcal{G}) \in \mathbb{R}^{m \times m}$ matrix (with $W=I_{m}$, where $I_{m}$ denotes the identity matrix of dimension $m$ ) is defined as

$$
\tilde{L}_{e}(\mathcal{G})=D(\mathcal{G})^{\top} D(\mathcal{G})
$$

Clearly, the graph Laplacian matrix is symmetric positive semi-definite; its eigenvalues are $\lambda_{n}(\mathcal{G}) \geq \cdots \geq \lambda_{2}(\mathcal{G}) \geq \lambda_{1}(\mathcal{G})=0$. The algebraic multiplicity of the zero eigenvalue of the graph Laplacian is equal to the number of connected components of the graph. For an undirected graph describing the connectivity of a network, say of single integrators, the second eigenvalue $\lambda_{2}(\mathcal{G})>0$ is known as the algebraic connectivity and it determines the convergence rate of the basic time-invariant consensus algorithm. For time-varying graphs, however, one cannot rely on eigen-value analysis to determine the rate of consensus convergence [7], [9]. In this paper, we present diverse exponential-convergence estimates for undirected graphs of single and doubleintegrators with time-varying interconnections. Our main statements rely on tools for stability analysis of linear time-varying systems and the following hypothesis.

Assumption 1. The topology of the time-varying graph $\mathcal{G}(t)$ is such that, if the weights matrix $W \equiv I_{m}$, then the graph is connected.

Assumption 1 does not state that the interconnections are constant nor that the graph is 
connected for each $t \in \mathbb{R}^{+}$but that the topology is such that if the interconnections were constant, the graph would be connected.

\section{B. Consensus of single-integrator agents}

Consider a set of $n$ vertices $v_{i}$, which represent single integrators, that is, for all $i \leq n$, we have

$$
\dot{x}_{i}=u_{i}, \quad x_{i} \in \mathbb{R}
$$

where $u_{i}$ denotes a control input. In the classical consensus paradigm this input is defined by

$$
u_{i}=-k \sum_{j=1}^{n} a_{i j}(t)\left(x_{i}-x_{j}\right) \quad j \neq i
$$

where $k>0$ is a scalar gain, $a_{i j}(t) \geq 0$ represents the strength of the influence of agent $j$ on agent $i$, for $i, j \leq n$. We assume that the interactions are symmetric, that is, $a_{i j}(t)=a_{j i}(t)$ for all $i, j \leq n$ hence, the graph satisfies is cut-balanced [10]. Now, substituting (3) in (2) and defining $x:=\left[x_{1}, \cdots, x_{n}\right]^{\top}$, we obtain

$$
\dot{x}=-k D(\mathcal{G}) W(t) D(\mathcal{G})^{\top} x
$$

To obtain the latter equation we have used the fact that the Laplacian matrix, which satisfies $L(\mathcal{G})=D(\mathcal{G}) W D(\mathcal{G})^{\top}$, may also be defined as $L:=\left[\ell_{i j}\right]$ where $\ell_{i j}=-a_{i j}$ for all $i \neq j$ and $\ell_{i i}:=\sum_{j=1, j \neq i}^{n} a_{i j}$ hence, $\dot{x}=-k L(\mathcal{G}) x$.

For further analysis, we employ the edge agreement transformation proposed in [7]. To that end, we recall the relationship between node and edge agreement protocol -see [13, pp. 77-81] and [7, equation 8]:

$$
x_{e}:=D(\mathcal{G})^{\top} x
$$

Hence, differentiating (5) and using (4) we obtain

$$
\dot{x}_{e}=-k \tilde{L}_{e}(\mathcal{G}) W(t) x_{e}
$$

On one hand, the transformation (5) makes it clear that the consensus analysis problem for equation (4) is equivalent to studying the convergence of the edge states $x_{e} \in \mathbb{R}^{m}$ to zero. 
On the other hand, it allows, under Assumption 1, to partition $x_{e}$ into states corresponding to spanning-tree edges, $x_{\tau} \in \mathbb{R}^{p}$, and states representing cycle edges, $x_{c} \in \mathbb{R}^{(m-p)}$ that is,

$$
x_{e}=\left[\begin{array}{l}
x_{\tau} \\
x_{c}
\end{array}\right] .
$$

Furthermore, under Assumption 1, we can arbitrarily fix one spanning tree. Then, $\mathcal{G}$ may be described as a union of two sub-graphs as $\mathcal{G}_{\tau} \cup \mathcal{G}_{c}$, where $\mathcal{G}_{\tau}$ and $\mathcal{G}_{c}$ represent the spanning tree and cycle edges of $\mathcal{G}$ respectively. Moreover, using an appropriate permutation of the edge-indices we can partition $D(\mathcal{G})$ as

$$
D(\mathcal{G})=\left[D\left(\mathcal{G}_{\tau}\right) \quad D\left(\mathcal{G}_{c}\right)\right]
$$

where, $D\left(\mathcal{G}_{\tau}\right) \in \mathbb{R}^{n \times p}$ and $D\left(\mathcal{G}_{c}\right) \in \mathbb{R}^{n \times(m-p)}$ represent respectively, the incidence matrix corresponding to spanning-tree and cycle edges. Therefore, in terms of the above permutations, the edge-Laplacian matrix $\tilde{L}_{e}(\mathcal{G})$ defined in (1) corresponds to

$$
\tilde{L}_{e}(\mathcal{G})=\left[\begin{array}{cc}
\tilde{L}_{e}\left(\mathcal{G}_{\tau}\right) & D\left(\mathcal{G}_{\tau}\right)^{\top} D\left(\mathcal{G}_{c}\right) \\
D\left(\mathcal{G}_{c}\right)^{\top} D\left(\mathcal{G}_{\tau}\right) & \tilde{L}_{e}\left(\mathcal{G}_{c}\right)
\end{array}\right]
$$

where $\tilde{L}_{e}\left(\mathcal{G}_{\tau}\right) \in \mathbb{R}^{p \times p}$ and $\tilde{L}_{e}\left(\mathcal{G}_{c}\right) \in \mathbb{R}^{(m-p) \times(m-p)}$ are used to denote the edge-Laplacian matrix corresponding to spanning tree and cycle edges. Likewise, $W(t) \in \mathbb{R}^{m \times m}$ may also be partitioned as

$$
W(t)=\left[\begin{array}{cc}
W_{\tau}(t) & 0 \\
0 & W_{c}(t)
\end{array}\right]
$$

where $W_{\tau}(t) \in \mathbb{R}^{p \times p}$ and $W_{c}(t) \in \mathbb{R}^{(m-p) \times(m-p)}$ denote the weight matrices corresponding to the spanning-tree and cycle edges, respectively. Furthermore, the columns of the cycle edges $D\left(\mathcal{G}_{c}\right) \in \mathbb{R}^{n \times(m-p)}$ are linearly dependent on the columns of $D\left(\mathcal{G}_{\tau}\right) \in \mathbb{R}^{n \times p}$. This relationship can be expressed as

$$
D\left(\mathcal{G}_{\tau}\right) Z=D\left(\mathcal{G}_{c}\right)
$$

where $Z \in \mathbb{R}^{p \times(m-p)}$ is defined as

$$
Z:=\left(D\left(\mathcal{G}_{\tau}\right)^{\top} D\left(\mathcal{G}_{\tau}\right)\right)^{-1} D\left(\mathcal{G}_{\tau}\right)^{\top} D\left(\mathcal{G}_{c}\right)
$$


Thus, substituting (9), (10) and (7) in (6) we obtain that the states corresponding to the spanningtree edges and to the cycle edges evolve according to

$$
\begin{aligned}
& \dot{x}_{\tau}=-k \tilde{L}_{e}\left(\mathcal{G}_{\tau}\right) W_{\tau}(t) x_{\tau}-k D\left(\mathcal{G}_{\tau}\right)^{\top} D\left(\mathcal{G}_{c}\right) W_{c}(t) x_{c} \\
& \dot{x}_{c}=-k D\left(\mathcal{G}_{c}\right)^{\top} D\left(\mathcal{G}_{\tau}\right) W_{\tau}(t) x_{\tau}-k \tilde{L}_{e}\left(\mathcal{G}_{c}\right) W_{c}(t) x_{c} .
\end{aligned}
$$

The subset of spanning-tree edges constitutes a minimal representation of the edge states in the sense that consensus is achieved if and only if $x_{\tau} \rightarrow 0$. Hence, we shall focus on the study of the $x_{\tau}$ dynamics. Indeed, the cycle-edges states can be reconstructed from the spanning-tree edge states as $x_{c}=Z^{\top} x_{\tau}$ hence, we see that (12) is equivalent to

$$
\dot{x}_{\tau}=-k \tilde{L}_{e}\left(\mathcal{G}_{\tau}\right) R W(t) R^{\top} x_{\tau}
$$

where, $R:=\left[\begin{array}{ll}I_{p} & Z\end{array}\right] \in \mathbb{R}^{p \times m}$. Now, since $\tilde{L}_{e}\left(\mathcal{G}_{\tau}\right) \in \mathbb{R}^{p \times p}$ is symmetric positive definite, it can be decomposed as $\tilde{L}_{e}\left(\mathcal{G}_{\tau}\right)=\Gamma \Lambda \Gamma^{\top}$, where $\Gamma \in \mathbb{R}^{p \times p}$ is a unitary matrix and $\Lambda \in \mathbb{R}^{p \times p}$ is the diagonal matrix resulting from the Jordan decomposition (and hence contains eigenvalues) of the spanning tree edge Laplacian. Using the latter decomposition in the spanning-tree equation (14) yields

$$
\dot{x}_{\tau}=-k \Gamma \Lambda \Gamma^{\top} R W(t) R^{\top} x_{\tau}
$$

and, using the similarity transformation $y:=\Gamma^{\top} x_{\tau}$, we obtain

$$
\dot{y}=-k \Lambda \Gamma^{\top} R W(t) R^{\top} \Gamma y
$$

Thus, we see that the networked systems achieve consensus if the origin of (15) is uniformly globally exponentially stable for the latter equation. Through our main results we establish the latter and, more significantly, explicit convergence rate bounds.

\section{Consensus in double-integrator dynamics}

We show now that, by applying the edge-agreement protocol [13], the consensus problem for networks of second-order systems may also be (mainly) recasted into the study of Equation (15). To that end, let us consider a network of second-order integrators,

$$
\dot{x}_{i}=y_{i}, \quad \dot{y}_{i}=u_{i}, \quad i \leq n
$$


under the action of the update algorithm

$$
u_{i}=u_{i_{1}}+u_{i_{2}}
$$

where

$$
u_{i_{1}}=-\beta y_{i}, \quad \beta>0
$$

is the local velocity feedback and

$$
u_{i_{2}}=-\alpha \sum_{j=1}^{n} a_{i j}(t)\left[x_{i}-x_{j}\right]-\gamma \sum_{j=1}^{n} a_{i j}(t)\left[y_{i}-y_{j}\right]
$$

where $\alpha>0$ and $\gamma=\alpha / \beta$ are positive scalar gains. Incorporating the control laws specified in (18) and (19), the closed loop dynamics becomes:

$$
\begin{aligned}
& \dot{x}=y \\
& \dot{y}=-\beta y-\alpha D(\mathcal{G}) W(t) D(\mathcal{G})^{\top} x-\gamma D(\mathcal{G}) W(t) D(\mathcal{G})^{\top} y
\end{aligned}
$$

where we used $L(\mathcal{G})=D(\mathcal{G}) W(t) D(\mathcal{G})^{\top}$. In order to convert the above consensus problem to a stabilization one we mimic the relationship between the edge and node agreement protocol as defined in [13, pp. 132-134]. Differentiating

$$
\begin{aligned}
& x_{e_{1}}:=D(\mathcal{G})^{\top} x \\
& x_{e_{2}}:=D(\mathcal{G})^{\top} y .
\end{aligned}
$$

results in

$$
\begin{aligned}
& \dot{x}_{e_{1}}=x_{e_{2}} \\
& \dot{x}_{e_{1}}=-\beta x_{e_{2}}-\alpha \tilde{L}_{e}(\mathcal{G}) W(t) x_{e_{1}}-\gamma \tilde{L}_{e}(\mathcal{G}) W(t) x_{e_{2}}
\end{aligned}
$$

Now, since the graph $\mathcal{G}$ with $W \equiv I_{m}$ is connected, it can be decomposed as $\mathcal{G}_{\tau} \cup \mathcal{G}_{c}$. Then, using an appropriate permutation of the edge indices, the edge state vector $\left(x_{e_{1}}, x_{e_{2}} \in \mathbb{R}^{m}\right)$ may be further subdivided into

$$
x_{e_{1}}=\left[\begin{array}{l}
x_{\tau_{1}} \\
x_{c_{1}}
\end{array}\right] \quad x_{e_{2}}=\left[\begin{array}{l}
x_{\tau_{2}} \\
x_{c_{2}}
\end{array}\right] .
$$

The symbols $x_{\tau_{1}}, x_{\tau_{2}} \in \mathbb{R}^{p}$ and $x_{c_{1}}, x_{c_{2}} \in \mathbb{R}^{m-p}$ denote the spanning-tree and the cycle edge states, respectively, corresponding to the position and velocity interaction topology associated 
with $x_{i}$ and $y_{i} \in \mathbb{R}$. The columns of the cycle edges $D\left(\mathcal{G}_{c}\right) \in \mathbb{R}^{n \times(m-p)}$ are linearly dependent on the columns of $D\left(\mathcal{G}_{\tau}\right) \in \mathbb{R}^{n \times p}$ that is,

$$
D\left(\mathcal{G}_{\tau}\right) Z=D\left(\mathcal{G}_{c}\right)
$$

where $Z \in \mathbb{R}^{p \times(m-p)}$ is defined in equation (11). Hence, expanding out (23) we obtain

$$
\begin{aligned}
& \dot{x}_{e_{2}}=-\beta x_{e_{2}} \\
&-\alpha {\left[\begin{array}{cc}
\tilde{L}_{e}\left(\mathcal{G}_{\tau}\right) & D\left(\mathcal{G}_{\tau}\right)^{\top} D\left(\mathcal{G}_{c}\right) \\
D\left(\mathcal{G}_{c}\right)^{\top} D\left(\mathcal{G}_{\tau}\right) & \tilde{L}_{e}\left(\mathcal{G}_{c}\right)
\end{array}\right]\left[\begin{array}{cc}
W_{\tau}(t) & 0 \\
0 & W_{c}(t)
\end{array}\right] x_{e_{1}} } \\
&-\gamma\left[\begin{array}{cc}
\tilde{L}_{e}\left(\mathcal{G}_{\tau}\right) & D\left(\mathcal{G}_{\tau}\right)^{\top} D\left(\mathcal{G}_{c}\right) \\
D\left(\mathcal{G}_{c}\right)^{\top} D\left(\mathcal{G}_{\tau}\right) & \tilde{L}_{e}\left(\mathcal{G}_{c}\right)
\end{array}\right]\left[\begin{array}{cc}
W_{\tau}(t) & 0 \\
0 & W_{c}(t)
\end{array}\right] x_{e_{2}} .
\end{aligned}
$$

Now, as in the single-integrator case, our main interest lies in the behavior of the spanning-tree edge dynamics. This is given by

$$
\begin{aligned}
& \dot{x}_{\tau_{1}}=x_{\tau_{2}} \\
& \begin{aligned}
\dot{x}_{\tau_{2}}=-\beta x_{\tau_{2}}-\alpha & \tilde{L}_{e}\left(\mathcal{G}_{\tau}\right)\left[W_{\tau}+Z W_{c} Z^{\top}\right] x_{\tau_{1}} \\
& \quad-\gamma \tilde{L}_{e}\left(\mathcal{G}_{\tau}\right)\left[W_{\tau}+Z W_{c} Z^{\top}\right] x_{\tau_{2}}
\end{aligned}
\end{aligned}
$$

Moreover, since $\tilde{L}_{e}\left(\mathcal{G}_{\tau}\right) \in \mathbb{R}^{p \times p}$ is symmetric and positive definite, it admits the Jordan decomposition $\tilde{L}_{e}\left(\mathcal{G}_{\tau}\right)=\Gamma \Lambda \Gamma^{\top}$. Using this in (26) we obtain

$$
\begin{aligned}
& \dot{x}_{\tau_{1}}=x_{\tau_{2}} \\
& \dot{x}_{\tau_{2}}=-\left[\beta+\gamma \Gamma \Lambda \Gamma^{\top} R W(t) R^{\top}\right] x_{\tau_{2}} \\
& \\
& \quad-\alpha \Gamma \Lambda \Gamma^{\top} R W(t) R^{\top} x_{\tau_{1}}
\end{aligned}
$$

Then, introducing the similarity transformation

$$
\left[\begin{array}{l}
z_{1} \\
z_{2}
\end{array}\right]=\left[\begin{array}{ll}
\Gamma^{\top} & 0 \\
0 & \Gamma^{\top}
\end{array}\right]\left[\begin{array}{l}
x_{\tau_{1}} \\
x_{\tau_{2}}
\end{array}\right],
$$

the spanning-tree edge dynamics becomes

$$
\begin{aligned}
\dot{z}_{1}= & z_{2} \\
\dot{z}_{2}= & -\left[\beta+\gamma \Lambda \Gamma^{\top} R W(t) R^{\top} \Gamma\right] z_{2} \\
& \quad-\alpha \Lambda \Gamma^{\top} R W(t) R^{\top} \Gamma z_{1} .
\end{aligned}
$$


From (28), since $\Gamma$ has full rank, we see that $\{z=0\}$ is uniformly globally exponentially stable for (29) if and only if so is $\left\{x_{\tau}=0\right\}$ for the system (27). Then, to establish exponential stability of $\{z=0\}$ we observe that, defining

$$
\zeta:=z_{2}+\beta z_{1}
$$

and recalling that $\beta:=\alpha / \gamma$, the system's dynamics (29) is equivalent to

$$
\begin{aligned}
\dot{z}_{1} & =-\beta z_{1}+\zeta \\
\dot{\zeta} & =-\gamma \Lambda \Gamma^{\top} R W(t) R^{\top} \Gamma \zeta .
\end{aligned}
$$

To establish uniform global exponential stability of the origin $\left\{\left(z_{1}, \zeta\right)=(0,0)\right\}$ for (30a)(30b) we can invoke a simple cascades argument. Indeed, $\left\{z_{1}=0\right\}$ is exponentially stable for $\dot{z}_{1}=-\beta z_{1}$ and (30a) is input to state stable with linear gain. It is only left to prove that $\{\zeta=0\}$ is uniformly globally exponentially stable for (30b) - cf. Eq. (15).

\section{CONVERGENCE ANALYSIS OF TIME-VARYING CONSENSUS PROTOCOLS}

In the previous sections we have showed that the consensus analysis problem, for the singleintegrator (respectively, for the double-integrator systems) boils down to a problem of exponential stability analysis of the origin of an equation of the form (15) (respectively Eq. (30b)). In this section we present our main results. We present several statements on uniform global exponential stability of the origin of (15) and give explicit exponential convergence estimates for the system's solutions that is, we establish explicit expressions for $\kappa$ and $\gamma$ in

$$
\left|y\left(t, t_{\circ}, y_{\circ}\right)\right| \leq \kappa\left|y_{\circ}\right| e^{-\gamma\left(t-t_{\circ}\right)} \quad \forall t \geq t_{\circ}, y\left(t_{\circ}\right):=y_{\circ}
$$

and all $\left(t, y_{\circ}\right) \in \mathbb{R}_{\geq 0} \times \mathbb{R}^{p}$.

Furthermore, we remark that the convergence rate $\gamma$ for the spanning-tree edges trajectories, $x_{\tau}(t)$, and for $y(t)$ as described in (31) are identical. To see this, note that $y(t)$ and $x_{\tau}(t)$ are related to each other via the similarity transformation $x_{\tau}(t):=\Gamma y(t)$ with orthogonal matrix $\Gamma$, a simple algebraic manipulation of (31) translates to

$$
\left|x_{\tau}(t)\right| \leq|\Gamma||y(t)| \leq \kappa|\Gamma|\left|y_{\circ}\right| e^{-\gamma\left(t-t_{\circ}\right)} \quad \forall t \geq t_{\circ} .
$$

Our statements rely on the Assumption 1 and on the following hypothesis concerning the spanning-tree edges interconnections matrix $W_{\tau}(t)$. 
Assumption 2. The function $W_{\tau}: \mathbb{R}_{\geq 0} \rightarrow \mathbb{R}^{p \times p}$ is persistently exciting that is, there exist $\mu>0$ and $T>0$ such that

$$
\int_{t}^{t+T} W_{\tau}(s) d s \geq \mu I_{p} \quad \forall t \geq 0 .
$$

It is important to emphasize that, since its first appearance in the literature of identification -see [3], depending of the context in which it is used, persistency of excitation has been (re)defined in various ways. In this paper we adopt the following.

Definition 1 (persistency of excitation). A locally integrable function $\phi: \mathbb{R}_{\geq 0} \rightarrow \mathbb{R}^{n \times m}$ is said to be persistently exciting if there exist positive constants $\mu_{1}$ and $T$ such that

$$
\int_{t}^{t+T} \phi(\sigma) \phi(\sigma)^{\top} d \sigma \geq \mu_{1} I_{n} \quad \forall t \geq 0 .
$$

However, persistency of excitation is sometimes defined as the property that, in addition to (33), there exist $\mu_{2}>0$ such that

$$
\mu_{2} I_{n} \geq \int_{t}^{t+T} \phi(\sigma) \phi(\sigma)^{\top} d \sigma \quad \forall t \geq 0 .
$$

Then, since at least [1], under either boundedness of $\phi$ or the condition (34), persistency of excitation is well known to be a necessary and sufficient condition for the origin of adaptive control systems to be uniformly globally exponentially stable. In particular, this is the case for the so-called gradient descent equation

$$
\dot{y}(t)=-\phi(t) \phi(t)^{\top} y(t)
$$

Furthermore, in more recent articles -see [5], [11] explicit exponential convergence rates, as a function of $\mu_{1}, \mu_{2}$, and $T$, are established. As we shall see, depending on the method of proof, the choice of the definition of persistency of excitation is not innocuous.

In this paper, we recast the consensus analysis problem for first and second order systems in that of the stability analysis of (15) and (30b) respectively. Thus, our main statements rely on the study of these equations or, actually, of (35) with $\phi(t):=\sqrt{k} \Gamma^{\top} R W(t)^{1 / 2}$. Indeed, note that if $W_{\tau}^{1 / 2}$ is persistently exciting (Assumption 2$)$ so is $\phi$ since $\Gamma$ is full column rank $(=p)$ and 
$W_{c} \geq 0$. Indeed, we have

$$
\begin{gathered}
\int_{t}^{t+T} \phi(s) \phi(s)^{\top} d s=\int_{t}^{t+T} k \Gamma^{\top} R W(s) R^{\top} \Gamma d s \\
=\int_{t}^{t+T} k \Gamma^{\top} W_{\tau}(s) \Gamma d s+k \Gamma^{\top} Z W_{c}(s) Z^{\top} \Gamma d s \\
\geq \mu k \lambda_{\min }\left(\Gamma^{\top} \Gamma\right)=: \mu_{1} .
\end{gathered}
$$

Whence Assumption 2, which is stated as a hypothesis on the spanning-tree interconnection weights matrix $W_{\tau}$.

For simplicity, in the sequel we set $\mathrm{k}=1$ in (15) and, to compact the notation, we define $\lambda_{m}:=\lambda_{\min }(\Lambda)$ and $\lambda_{M}:=\lambda_{\max }(\Lambda)$. Our first statement is based on [1] and it was proved in [7].

Theorem 1 (A-estimate). Let Assumptions 1 and 2 hold. Further assume that there exists $\mu_{2}>0$ such that (34) holds. Then, (31) holds with

$$
\begin{gathered}
\gamma_{A}=-\frac{1}{2 T} \ln \left[1-\frac{2 \lambda_{m} \mu_{1}}{\left(1+\lambda_{M} \sqrt{p} \mu_{2}\right)^{2}}\right], \\
\kappa=\left[\frac{\lambda_{M} m_{v}}{\lambda_{m}}\right]^{1 / 2}, \quad m_{v}:=\frac{1}{\left[1-\frac{2 \lambda_{m} \mu_{1}}{\left(1+\lambda_{M} \sqrt{p} \mu_{2}\right)^{2}}\right]} .
\end{gathered}
$$

Notice the dependence of the convergence estimate $\gamma_{A}$ on the system's dimension, $p$. As the latter increases, the rate of convergence decreases.

Our second statement follows the proof-lines in [11].

Theorem 2. (LP-estimate) Assume that $W(t)$ is uniformly bounded and Assumptions 1 and 2 hold. Then, (31) holds with $\kappa=\sqrt{\lambda_{M} / \lambda_{m}}$ and $\gamma=\gamma_{L P}$ where ${ }^{1}$

$$
\gamma_{L P}=-\frac{1}{2 T} \ln \left[1-\frac{2 \lambda_{m} \mu_{1}}{\left[1+\lambda_{M} \delta_{T}\right]^{2}}\right] \text {. }
$$

where,

$$
\delta_{T}:=T\left|\Gamma^{\top} R W(t) R^{\top} \Gamma\right|_{\infty}
$$

${ }^{1}$ For a function $F: \mathbb{R}_{\geq 0} \rightarrow \mathbb{R}^{p \times p}$ we use the notation $\|F\|_{\infty}:=\sup _{t \geq 0}|F(t)|$ where $|F(t)|$ denotes the induced Euclidean matrix norm. 
Remark 1. Note that the stability estimates given by Theorems 1 and 2 are quite similar, even though these statements rely on different conditions. In the former it is assumed that the $\phi$ is locally integrable -see (34)- while in the latter, it is $\phi$ which is assumed to be bounded. If we set $\mu=\delta_{T}$ and, to avoid the unnecessary conservatism in $\gamma_{A}$, we set $p=1$, both decay rates are equivalent.

Proof. Consider the Lyapunov function candidate $V_{1}: \mathbb{R}^{p} \rightarrow \mathbb{R}_{\geq 0}$, defined as

$$
V_{1}(y)=\frac{1}{2} y^{\top} \Lambda^{-1} y
$$

whose total derivative along the trajectories of (15) (with $k=1$ ) satisfies

$$
\dot{V}_{1}(y(t))=-y(t)^{\top} \Gamma^{\top} R W(t) R^{\top} \Gamma y(t) \leq 0
$$

Now, integrating (40) over the interval $[t, t+T]$, we get:

$$
V_{1}(y(t+T))-V_{1}(y(t))=-\int_{t}^{t+T}\left|W(s)^{1 / 2} R^{\top} \Gamma y(s)\right|^{2} d s
$$

On the other hand, solving (15), we have

$$
y(s)=y(t)-\Lambda \int_{t}^{s} \Gamma^{\top} R W(\tau) R^{\top} \Gamma y(\tau) d \tau .
$$

Hence, substituting the later in (41) we obtain

$$
\begin{array}{r}
V_{1}(y(t+T))-V_{1}(y(t))=-\int_{t}^{t+T} \mid W(s)^{1 / 2} R^{\top} \Gamma y(t) \\
-\left.W(s)^{1 / 2} R^{\top} \Gamma \Lambda \int_{t}^{s} \Gamma^{\top} R W(\tau) R^{\top} \Gamma y(\tau) d \tau\right|^{2} d s .
\end{array}
$$

Next, we use the inequality

$$
(a-b)^{2} \geq \frac{\rho}{1+\rho} a^{2}-\rho b^{2}
$$

which holds for any $a, b \in \mathbb{R}$ and $\rho>0$, to obtain

$$
\begin{aligned}
& V_{1}(y(t+T))-V_{1}(y(t)) \leq \\
& \quad-\frac{\rho}{1+\rho}|y(t)|^{2} \int_{t}^{t+T}\left|W(s)^{1 / 2} R^{\top} \Gamma\right| d s \\
& \quad+\rho \int_{t}^{t+T}\left|W(s)^{1 / 2} R^{\top} \Gamma \Lambda \int_{t}^{s} \Gamma^{\top} R W(\tau) R^{\top} \Gamma y(\tau) d \tau\right|^{2} d s .
\end{aligned}
$$


Now, let the condition of persistency of excitation on $W_{\tau}(t)$ generate $\mu_{1}>0$ such that (36) holds with $\phi(t):=\Gamma^{\top} R W(t)^{1 / 2}$. Then, the first term on the right hand side of (44) satisfies,

$$
-\frac{\rho|y(t)|^{2}}{1+\rho} \int_{t}^{t+T}\left|W(s)^{1 / 2} R^{\top} \Gamma\right|^{2} d s \leq-\frac{\rho \mu_{1}}{1+\rho}|y(t)|^{2} .
$$

Furthermore, using the Cauchy-Schwartz inequality, we see that the second term satisfies the following:

$$
\begin{gathered}
\int_{t}^{t+T}\left|W(s)^{1 / 2} R^{\top} \Gamma \Lambda \int_{t}^{s} \Gamma^{\top} R W(\tau) R^{\top} \Gamma y(\tau) d \tau\right|^{2} d s \\
=\int_{t}^{t+T} \mid \int_{t}^{s}\left[W(s)^{1 / 2} R^{\top} \Gamma \Lambda \Gamma^{\top} R W^{1 / 2}(\tau)\right] \\
\times\left.\left[W^{1 / 2}(\tau) R^{\top} \Gamma y(\tau)\right] d \tau\right|^{2} d s \\
\leq \int_{t}^{t+T}\left[\int_{t}^{s}\left|W(s)^{1 / 2} R^{\top} \Gamma \Lambda \Gamma^{\top} R W^{1 / 2}(\tau)\right|^{2} d \tau\right] \\
\left.\times \int_{s \in[t, t+T]}^{s}\left|W(\tau)^{1 / 2} R^{\top} \Gamma y(\tau)\right|^{2} d \tau\right] d s \\
\left.\leq \int_{t}^{s}\left|W(s)^{1 / 2} R^{\top} \Gamma \Lambda \Gamma^{\top} R W^{1 / 2}(\tau)\right|^{2} d \tau\right] \\
\times\left[\int_{t}^{s}\left|W(\tau)^{1 / 2} R^{\top} \Gamma y(\tau)\right|^{2} d \tau\right] \\
=\int_{t}^{t+T}\left|W(s)^{1 / 2} R^{\top} \Gamma \Lambda \Gamma^{\top} R W^{1 / 2}(\tau)\right|^{2} d \tau \\
\times \int_{t}^{t+T}\left|W^{1 / 2}(\tau) R^{\top} \Gamma y(\tau)\right|^{2} d \tau \\
\leq T^{2} \lambda_{M}^{2}\left|\Gamma^{\top} R W(t) R^{\top} \Gamma\right|_{\infty}^{2}\left(V_{1}(y(t))-V_{1}(y(t+T))\right) .
\end{gathered}
$$

Thus, using the latter, (45) and $|y(t)|^{2} \geq 2 \lambda_{m} V_{1}(y(t))$ in (44) we obtain

$$
\begin{array}{r}
\frac{2 \rho \lambda_{m} \mu_{1}}{(1+\rho)} V_{1}(y(t)) \leq\left[\rho T^{2} \lambda_{M}^{2}\left|\Gamma^{\top} R W(t) R^{\top} \Gamma\right|_{\infty}^{2}+1\right] \\
\times\left[V_{1}(y(t))-V_{1}(y(t+T))\right]
\end{array}
$$

which, in turn, implies that

$$
\begin{aligned}
& V_{1}(y(t+T)) \leq \\
& \quad\left[1-\frac{2 \lambda_{m} \rho \mu_{1}}{(1+\rho)\left(\rho T^{2} \lambda_{M}^{2}\left|\Gamma^{\top} R W(t) R^{\top}\right|_{\infty}^{2}+1\right)}\right] V_{1}(y(t)) .
\end{aligned}
$$


Hence, defining

$$
\rho:=\left[T \lambda_{M}\left|\Gamma^{\top} R W(t) R^{\top} \Gamma\right|_{\infty}\right]^{-1}
$$

we obtain $V_{1}(y(t+T)) \leq \eta V_{1}(y(t)$ where

$$
\eta:=\left[1-\frac{2 \lambda_{m} \mu_{1}}{\left[T \lambda_{M}\left|\Gamma^{\top} R W(t) R^{\top} \Gamma\right|_{\infty}+1\right]^{2}}\right]
$$

so, setting $e^{-\gamma_{L P} T}=\eta$ with $\gamma_{L P}$ as in (38) and performing a simple computation, we find that $y(t)$ satisfies (31) with $\gamma=\gamma_{L P}$ and $\kappa=\sqrt{\lambda_{M} / \lambda_{m}}$.

Interestingly enough, if we reconsider the A-estimate obtained using the method of proof of [1] — cf. [7], considering the boundedness of $\Gamma^{\top} R W(t) R^{\top} \Gamma$ instead of (34) we may recover the same convergence rate as in Theorem 2 that is, $\gamma_{L P}=\gamma_{A}$. In other words, the convergence estimate that stems from [1] seems more conservative since the bound

$$
\begin{aligned}
\int_{t}^{t+T}\left|\Gamma^{\top} R W(s) R^{\top} \Gamma\right| d s \leq & \operatorname{tr}\left(\int_{t}^{t+T} \Gamma^{\top} R W(s) R^{\top} \Gamma d s\right) \\
& \leq p \mu_{2}
\end{aligned}
$$

which is proportional to the number of agents in the spanning-tree, is used. See [7] for details.

Our next statement follows the method of proof of stability of [5] for the gradient system (35).

Theorem 3. (B-Estimate) Let Assumptions 1-2 hold and $\phi(t):=\Gamma^{\top} R W(t)^{1 / 2}$ satisfy inequality (34). Then, the origin of (15) is uniformly globally exponentially stable and the solutions satisfy (31) with $\kappa=\sqrt{\lambda_{M} / \lambda_{m}}$ and $\gamma=\gamma_{B}$ where

$$
\gamma_{B}=-\frac{1}{2 T} \ln \left(1-2\left[-a+\sqrt{b+a^{2}}\right]^{2} \lambda_{m}\right)
$$

where

$$
a:=\frac{p^{1 / 2} \mu_{2}^{3 / 2} \lambda_{M}^{2}}{\lambda_{m} \sqrt{2}\left(1+2 \mu_{1} \lambda_{m}\right)}, \quad b:=\frac{\mu_{1} \lambda_{m}}{\lambda_{M}\left[1+2 \mu_{1} \lambda_{m}\right]} .
$$

Remark 2. The dependence of a on $p$, above, is to be noted. We remark that when $p \rightarrow \infty$, the argument of $\ln (\cdot)$ in (48) tends to 1 that is, the convergence rate diminishes as the size of the graph (number of agents) grows. The same holds for the A-estimate, in (37). The conservatism of these bounds comes from the fact that in Theorems 1 and 3 it is not assumed that $W$ is bounded, 
as in Theorems 2 and 4 further below, but, instead, the less restrictive local integrability bound (34) is imposed.

In that regard, it is not difficult to see that the A and LP estimates are of the same order if, in Theorem 1, it is assumed that $W(t)$ is bounded. Then, (34) holds with $\mu_{2}:=T|W(t)|_{\infty}$, for any $p$. The B-estimate, however, remains more conservative even if we impose boundedness on $W(t)$. This is further illustrated in numerical simulations in the next section.

Proof. Define $M: \mathbb{R}_{\geq 0} \rightarrow \mathbb{R}^{p \times p}$ as

$$
M(t):=\int_{0}^{t} \Gamma^{\top} R W(\sigma) R^{\top} \Gamma d \sigma \quad \forall t \geq 0 .
$$

Since $\Gamma \in \mathbb{R}^{p \times p}$ is full column rank, $W_{\tau}(t)$ is persistently exciting, and $\phi(t)$ satisfies (34), we have

$$
0<\mu_{1} I_{p} \leq M(T) \leq \mu_{2} I_{p}<\infty
$$

If necessary, after redefining $\mu_{1}$ and $\mu_{2}$. Next, consider the function $V: \mathbb{R}_{\geq 0} \times \mathbb{R}^{p} \rightarrow \mathbb{R}$ defined as

$$
V_{2}(t, y)=y^{\top} M(t) y+\frac{1}{2} y^{\top} \Lambda^{-1} y
$$

which is positive definite. Its total derivative along the system's trajectories satisfies

$$
\dot{V}_{2}(t, y(t))=-2 y(t)^{\top} \Gamma^{\top} R W(t) R^{\top} \Gamma \Lambda M(t) y(t) .
$$

Now, integrating on both sides of this equation, from 0 to $T$, we obtain

$$
\begin{aligned}
y(T)^{\top} & M(T) y(T)+V_{1}(y(T))-V_{1}(y(0)) \\
\leq & \left|2 \int_{0}^{T} y(\sigma)^{\top} \Gamma^{\top} R W(\sigma) R^{\top} \Gamma \Lambda M(\sigma) y(\sigma) d \sigma\right|
\end{aligned}
$$

-see (39). Now, since $W(t)$ is non-negative, the term on the right-hand side of (52) satisfies, in view of Cauchy-Schwartz inequality,

$$
\begin{aligned}
\left|2 \int_{0}^{T}\left[W(\sigma)^{1 / 2} R^{\top} \Gamma y(\sigma)\right]^{\top}\left[W(\sigma)^{1 / 2} R^{\top} \Gamma \Lambda M(\sigma) y(\sigma)\right] d \sigma\right| \\
\leq\left[2 \int_{0}^{T}\left|W(\sigma)^{1 / 2} R^{\top} \Gamma y(\sigma)\right|^{2} d \sigma\right]^{1 / 2} \\
\times\left[\int_{0}^{T}\left|W(\sigma)^{1 / 2} R^{\top} \Gamma \Lambda M(\sigma) y(\sigma)\right|^{2} d \sigma\right]^{1 / 2}
\end{aligned}
$$


We proceed to compute an upper-bound on the right-hand side of the inequality above. To that end, we analyze each of the terms in brackets separately. For the first, in view of (40), we have

$$
\int_{0}^{T}\left|W(\sigma)^{1 / 2} R^{\top} \Gamma y(\sigma)\right|^{2} d \sigma=V_{1}(y(0))-V_{1}(y(T)) .
$$

On the other hand, in view of (39) and (40) we have $|y(t)| \leq\left(\lambda_{M} / \lambda_{m}\right)|y(0)|$ therefore, the second factor can be bounded above by

$$
\begin{aligned}
& {\left[\int_{0}^{T}\left|W(\sigma)^{1 / 2} R^{\top} \Gamma \Lambda M(\sigma) y(\sigma)\right|^{2} d \sigma\right]^{1 / 2}} \\
& \quad \leq \frac{\lambda_{M}^{2}}{\lambda_{m}}\left[\int_{0}^{T}\left|W(\sigma)^{1 / 2} R^{\top} \Gamma\right|^{2}|M(\sigma)|^{2} d \sigma\right]^{1 / 2}|y(0)| .
\end{aligned}
$$

By assumption, $M(T)$ is bounded by $\mu_{2} I_{p}$ hence, $|M(T)| \leq \mu_{2}$. Also, $\left|W(\sigma)^{1 / 2} R^{\top} \Gamma\right|^{2}=$ $\left|\Gamma^{\top} R W(\sigma) R^{\top} \Gamma\right|$ hence, in view of (50) and (51) we have

$$
\left[\int_{0}^{T}\left|W(\sigma)^{1 / 2} R^{\top} \Gamma \Lambda M(\sigma) y(\sigma)\right|^{2} d \sigma\right]^{1 / 2} \leq \frac{\lambda_{M}^{2}}{\lambda_{m}}\left(p \mu_{2}^{3}\right)^{1 / 2}|y(0)|
$$

Then, putting together (52), (53), (54) and (56) yields,

$$
\begin{aligned}
y(T)^{\top} & M(T) y(T)-V_{1}(y(0))+V_{1}(y(T)) \\
& \leq p \mu_{2}^{3 / 2} \lambda_{M}|y(0)| \sqrt{2}\left[V_{1}(y(0))-V_{1}(y(T))\right]^{1 / 2} .
\end{aligned}
$$

Now, to compact the notation, let $f(s):=\left[V_{1}(y(0))-V_{1}(y(s))\right]^{1 / 2}$ where $V_{1}$ is defined in (39). Then, from

$$
\frac{1}{\lambda_{M}}|y|^{2} \leq y^{\top} \Lambda^{-1} y \leq \frac{1}{\lambda_{m}}|y|^{2}
$$

and (51), we have $y(T)^{\top} M(T) y(T) \geq 2 \mu_{1} \lambda_{m} V_{1}(y(T)) \geq 2 \mu_{1}\left[V_{1}(y(0))-f(T)^{2}\right]$. Replacing the latter in (57) and rearranging the terms we see that the latter inequality is equivalent to

$$
f(T)^{2}+\frac{\sqrt{2} p^{1 / 2} \mu_{2}^{3 / 2} \lambda_{M}^{2}|y(0)|}{\lambda_{m}\left(1+2 \mu_{1} \lambda_{m}\right)} f(T) \geq \frac{\mu_{1} \lambda_{m}|y(0)|^{2}}{\lambda_{M}\left[1+2 \mu_{1} \lambda_{m}\right]} .
$$

Solving (59) for $f(T)$ and using (49) we obtain

$$
f(T) \geq\left[-a+\sqrt{b+a^{2}}\right]|y(0)|
$$

which, in view of the definition of $f$ and (39), is equivalent to

$$
\frac{1}{2}\left[y(0)^{\top} \Lambda^{-1} y(0)-y(T)^{\top} \Lambda^{-1} y(T)\right] \geq\left[-a+\sqrt{b+a^{2}}\right]^{2}|y(0)|^{2}
$$


and, after some algebraic manipulations in which we use (58), we obtain

$$
|y(T)| \leq\left[\frac{\lambda_{M}}{\lambda_{m}}\right]^{1 / 2}\left[1-2\left[-a+\sqrt{b+a^{2}}\right]^{2} \lambda_{m}\right]^{1 / 2}|y(0)|
$$

Thus, setting, for any $T>0$,

$$
e^{-\gamma_{B} T}:=\left[1-2\left[-a+\sqrt{b+a^{2}}\right]^{2} \lambda_{m}\right]^{1 / 2}
$$

we obtain (48) and (31) holds on $[0, T]$. The interval may be extended to infinity by resetting the initial time and the initial conditions.

Hence by a simple algebraic manipulation we can conclude that $y(t)$ satisfies (31) with $\gamma=\gamma_{B}$ and $\kappa=\sqrt{\lambda_{M} / \lambda_{m}}$.

Our last statement on uniform global exponential stability for the speed-gradient system has the merit of relying on Lyapunov's direct method in addition to providing a stability estimate.

Theorem 4. Let Assumptions 1-2 hold and assume that there exists $\mu_{2}>0$ such that (34) holds. Then, (15) admits the Lyapunov function

$$
V(t, y)=\frac{1}{2} y^{\top}\left[\pi \Lambda^{-1}+Q(t)\right] y
$$

where

$$
\begin{aligned}
Q(t):=2 \delta_{T} I_{p}-\frac{2}{T} \int_{t}^{t+T} \int_{t}^{m} \Gamma^{\top} R W(s) R^{\top} \Gamma d s d m, \\
\delta_{T}:=T\left|\Gamma^{\top} R W(t) R^{\top} \Gamma\right|_{\infty}, \\
\pi:=1+\frac{2 \lambda_{M}^{2} \delta_{T}^{3}}{\mu_{1}} .
\end{aligned}
$$

Therefore, the solutions $y(t)$ satisfy (31) with

$$
\kappa=\left[\frac{\pi / \lambda_{m}+2 \delta_{T}}{\pi / \lambda_{M}}\right]^{1 / 2}
$$

and $\gamma=\gamma_{M}$, where

$$
\gamma_{M}:=\frac{\mu_{1}^{2} \lambda_{m}}{2 \delta_{T} T\left[\lambda_{M}^{2} \delta_{T}^{2}+\lambda_{m} \mu_{1}\right]+T \mu_{1}}
$$

Proof. We start by observing that

$$
0 \leq Q(t) \leq 2 \delta_{T} I_{p}
$$


therefore,

$$
\frac{\pi}{2 \lambda_{M}}|y|^{2} \leq V(t, y) \leq \frac{1}{2}\left[\frac{\pi}{\lambda_{m}}+2 \delta_{T}\right]|y|^{2} .
$$

Next, in view of the fundamental theorem of calculus, we obtain

$$
\dot{Q}(t)=-\frac{2}{T} \int_{t}^{t+T} \Gamma^{\top} R W(s) R^{\top} \Gamma d s+2 \Gamma^{\top} R W(t) R^{\top} \Gamma
$$

Then, let the condition of persistency of excitation on $W_{\tau}$ generate $\mu_{1}>0$ such that (36) holds with $k=1$. Using this and (64), we see that the total derivative of the Lyapunov function $V$ along trajectories of (15) satisfies

$$
\begin{aligned}
\dot{V}(t, y)=- & \pi y^{\top} \Gamma^{\top} R W(t) R^{\top} \Gamma y+y^{\top} \Gamma^{\top} R W(t) R^{\top} \Gamma \Lambda Q y \\
& -\frac{1}{T} y^{\top}\left[\int_{t}^{t+T} \Gamma^{\top} R W(s) R^{\top} \Gamma d s\right] y \\
& +y^{\top} \Gamma^{\top} R W(t) R^{\top} \Gamma y \\
\leq- & {[\pi-1] y^{\top} \Gamma^{\top} R W(t) R^{\top} \Gamma y-\frac{\mu_{1}}{T}|y|^{2} } \\
\leq- & +y^{\top} \Gamma^{\top} R W(t) R^{\top} \Gamma \Lambda Q y \\
& -\frac{\mu_{1}}{T}|y|^{2}+\frac{\epsilon}{2}\left|Q \Lambda \Gamma^{\top} R W(t)^{1 / 2}\right|^{2}|y|^{2} \\
\leq- & \frac{\mu_{1}}{2 T}|y|^{2}-\left[\pi-1-\frac{1}{2 \epsilon}\right]\left|W(t)^{1 / 2} R^{\top} \Gamma y\right|^{2} \\
& \quad-\left[\frac{\mu_{1}}{2 T}-\frac{\epsilon}{2}\left|Q \Lambda \Gamma^{\top} R W(t)^{1 / 2}\right|^{2}\right]|y|^{2} .
\end{aligned}
$$

Therefore, setting

$$
\epsilon:=\frac{\mu_{1}}{4 \delta_{T}^{3} \lambda_{M}^{2}}
$$

and using (61c), we obtain

$$
\dot{V}(t, y) \leq-\frac{\mu_{1}}{2 T}|y|^{2}
$$

which, in view of (63) implies that

$$
|y|^{2} \geq \frac{2 \lambda_{m} V(t, y)}{\pi+2 \lambda_{m} \delta_{T}}
$$

Therefore,

$$
\dot{V}(t, y) \leq-\frac{\mu_{1} \lambda_{m}}{\pi T+2 \lambda_{m} \delta_{T} T} V(t, y)
$$


and, replacing $\pi$ from (61c) we obtain

$$
\dot{V}(t, y) \leq-\gamma_{M} V(t, y)
$$

where $\gamma_{M}$ is defined in (62). The result follows integrating on both sides of the latter and using (63).

A simple inspection shows that $\gamma_{L P} \geq \gamma_{M}$ that is, the method of proof in [11] leads to a tighter estimate of the rate of convergence. However, the indisputable advantage of Theorem 4 is that it provides a strict Lyapunov function and a direct proof. This facilitates Lyapunov redesign as well as analysis of consensus of networked systems with drifts since, in contrast to the case of other statements, one does not need to rely on converse theorems.

\section{NumericAl Simulations}

\section{A. Examples for time-varying Consensus Protocols}

To illustrate our theoretical findings we have performed a series of numerical simulations. First, we consider a multi-agent system with five agents with single-integrator dynamics -see Eq. (16). The corresponding network graph (with arbitrary orientation) for the above multi-agent system is shown in Fig. 1 with $w_{i}(t)$ representing the weights corresponding to edge $e_{i}$. The

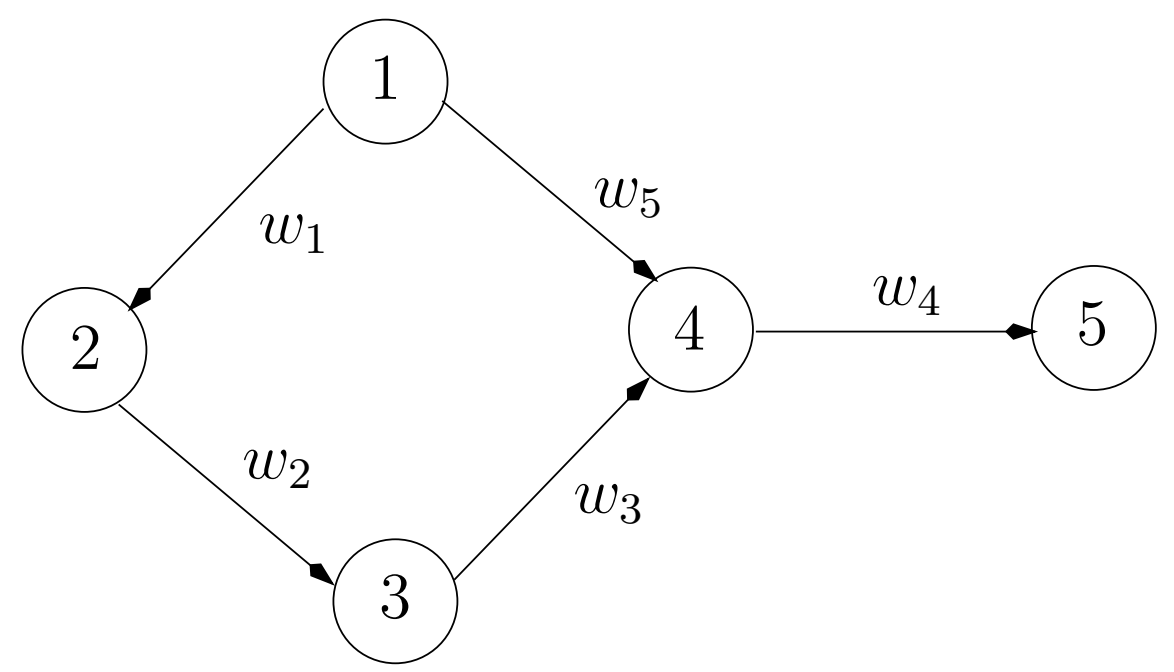

Fig. 1: Interaction graph $\left(\mathcal{G}^{\mathcal{O}}\right)$ between five agents 
incidence matrix, as defined in Section II-A, is

$$
D(\mathcal{G})=\left[\begin{array}{ccccc}
-1 & 0 & 0 & 0 & -1 \\
1 & -1 & 0 & 0 & 0 \\
0 & 1 & -1 & 0 & 0 \\
0 & 0 & 1 & -1 & 1 \\
0 & 0 & 0 & 1 & 0
\end{array}\right]
$$

The weight matrix is chosen as $W(t)=\operatorname{diag}\left[w_{1}(t), w_{2}(t), w_{3}(t), w_{4}(t), w_{5}(t)\right]$ where $w_{i}(t)=$ $\left\{\right.$ square $\left.\left(8\left(t-d_{i}\right)\right)+1\right\} \sin (i t)^{2}$ for $i=\{1,2,3,4,5\}$ with a duty cycle of 0.2 and time shift $d_{i}=0,0.157,0.316,0.4724,0.62$ seconds respectively. The initial conditions are set to $x_{0}=[0.1,0.2,0.4,0.7,1.1]^{\top}$. The results obtained from the simulations (for $k=1$ ) are shown in Fig. 2 from which one can appreciate the exponential rate convergence.

Furthermore, in order to illustrate the statements for the double-integrator case, we consider the double integrator agent dynamics (16) with the identical incidence $D(\mathcal{G})$ and weight matrix $W(t)$. The initial position and velocity coordinates are specified as $x_{0}=[0.2,0.4,0.6,0.8,1.0]^{\top}$ and $y_{0}=[0.2,0.4,0.6,0.8,1.0]^{\top}$. The design parameters are chosen as $\beta=\gamma=\alpha=1$. The simulation results for the same is given in, Figs. 3 and 4.

The control laws defined in (3) and (17) direct the five agents with single and double integrator dynamics, to move from their initial locations to the consensus value shown in Fig. 2 (for single integrator system) and Fig 3, Fig. 4 (for double integrator system).

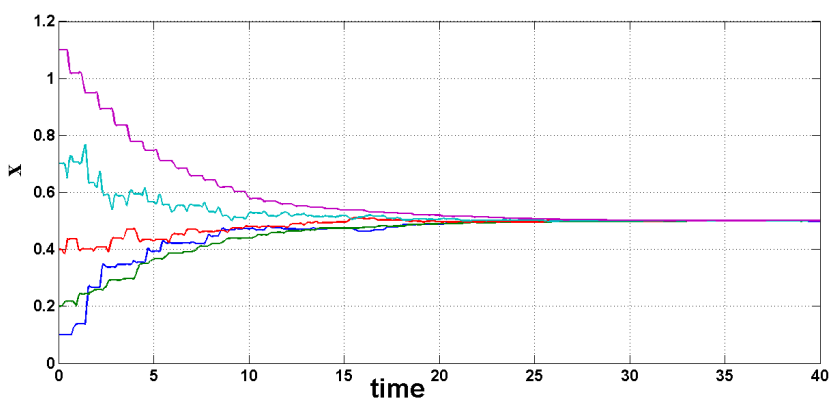

Fig. 2: Resulting state trajectories of the five single-intergrator agents

\section{B. Case studies to compare convergence estimates}

For the sake of comparison, we have evaluated numerically the convergence estimates in function of the parameters $\mu_{1}, \mu_{2}$ and $p$. A comparative study of the convergence rates $\gamma_{A}$, 


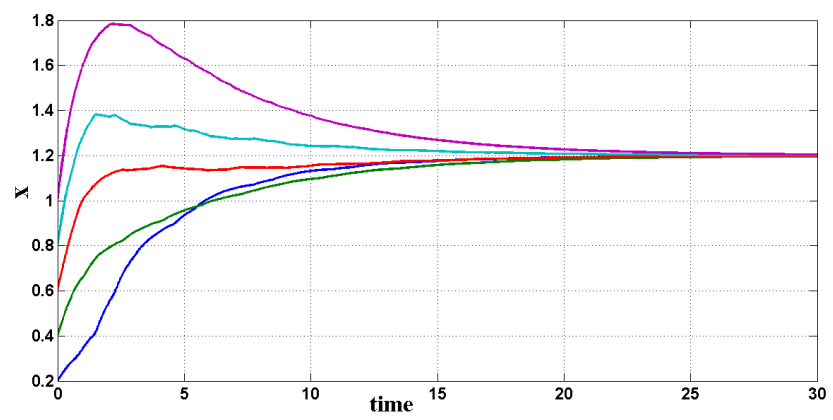

Fig. 3: Resulting position trajectories of the five double-intergrator agents

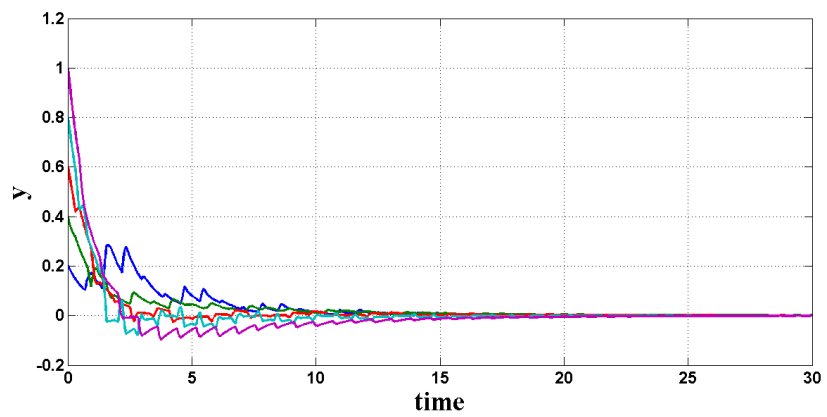

Fig. 4: Resulting velocity trajectories of the five agents

based on [1], and $\gamma_{B}$, based on [5], is documented in [2] for the case of a single agent (i.e., $p=n-1=0$ ). For the sake of comparison, consider (37) and let us define

$$
\eta_{A}:=\frac{2 \lambda_{m} \mu_{1}}{\left(1+\lambda_{M} \sqrt{p} \mu_{2}\right)^{2}} .
$$

Then, from (48) we have

$$
\begin{array}{r}
\gamma_{B}=-\frac{1}{2 T} \ln \left(1-\eta_{A}\right)-\frac{1}{2 T} \ln \left(J\left(\mu_{1}, \mu_{2}, p\right)\right) \\
J\left(\mu_{1}, \mu_{2}, p\right):=\frac{1-2 \lambda_{m}\left[-a+\sqrt{b+a^{2}}\right]^{2}}{1-\eta_{A}}
\end{array}
$$

It follows that $J\left(\mu_{1}, \mu_{2}, p\right)>1$ implies that $\gamma_{B}<\gamma_{A}$ and vice versa. Indeed, this is confirmed by our numerical evaluation which shows that the estimate in (37) is tighter.

In subsequent numerical evaluations we compare the A-estimate, $\gamma_{A}$, and the B-estimate, $\gamma_{B}$, for the single integrator multi-agent system. We reconsider the single-integrator agent dynamics (2) with the interaction graph given in Fig. 5. 


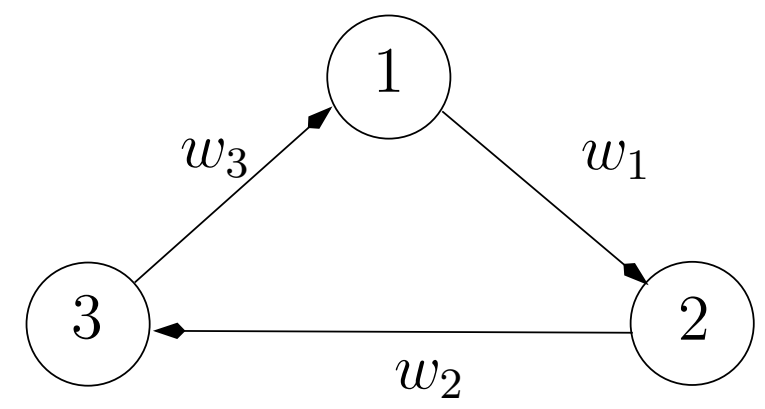

Fig. 5: Interaction graph $\left(\mathcal{G}^{\circ}\right)$ between three agents

The minimum and the maximum spanning tree eigenvalue for the afore-mentioned graph is given as $\lambda_{m}=1$ and $\lambda_{M}=3$ respectively. In Fig. 6 we represent the plots of $J\left(p, \mu_{1}, \mu_{2}\right)$ versus $\mu_{1}$ and different values of $\mu_{2}$ corresponding to $p=2$. Since, $\mu_{2} \geq \mu_{1}$, each curve extends only up to $\mu_{1}=\mu_{2}$. From the above simulations it can be conclude that $\gamma_{A}$, based on [1], corresponds to a tighter bound than that obtained with $\gamma_{B}$, computed based on [5]. Next, we present one

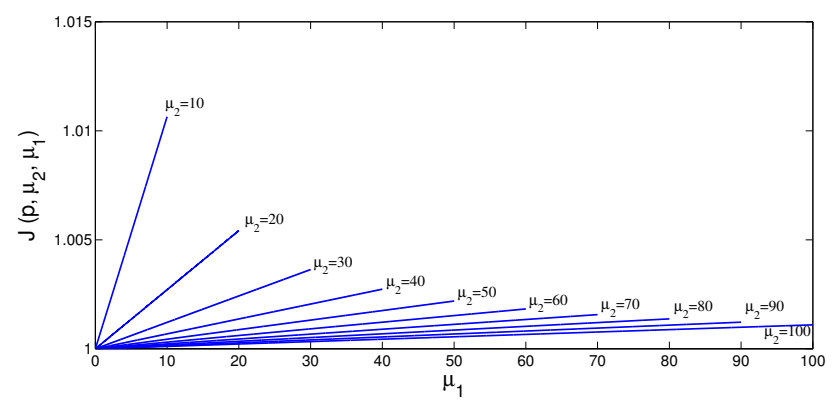

Fig. 6: Analysis of $J\left(p, \mu_{2}, \mu_{1}\right)$ for the graph in Fig. 5

case-study to compare the LP-estimate, $\gamma_{L P}$, and the estimate due to the Lyapunov function construction, $\gamma_{M}$ (based on Theorem 4). We reconsider the single-integrator agent dynamics with the interaction graph given in Fig 5.

In Fig. 7 we represent the plot of $J\left(\mu_{1}, \delta_{T}\right):=e^{2\left(T \gamma_{L P}-T \gamma_{M}\right)}$ versus $\mu_{1}$ and different values of $\delta_{T}$. Since, $\delta_{T} \geq \mu_{1}$, each curve extends only up to $\mu_{1}=\delta_{T}$. From the above simulations it can be conclude that, the LP-estimate $\gamma_{L P}$ gives a tighter bound than the one due to Lyapunov function construction $\gamma_{M}$ in the above case.

Finally, we present two case-studies to compare the $B$-estimate, $\gamma_{B}$, with respect to the $L P$ - 


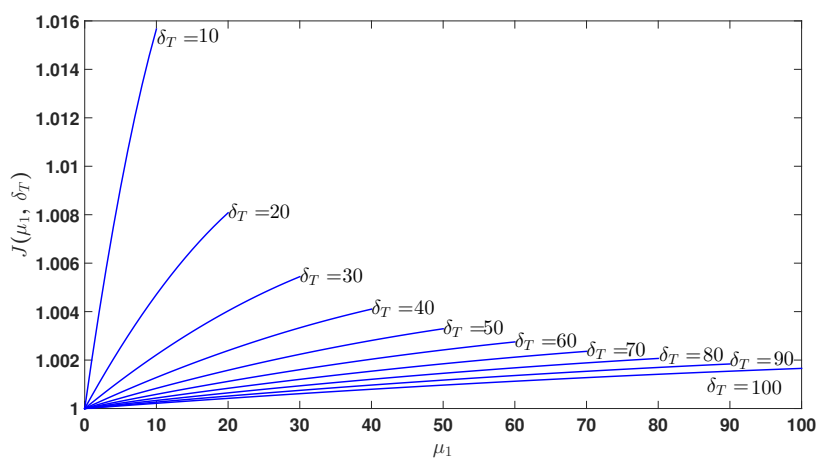

Fig. 7: Analysis of $J\left(\mu_{1}, \delta_{T}\right)$ for the graph in Fig. 5

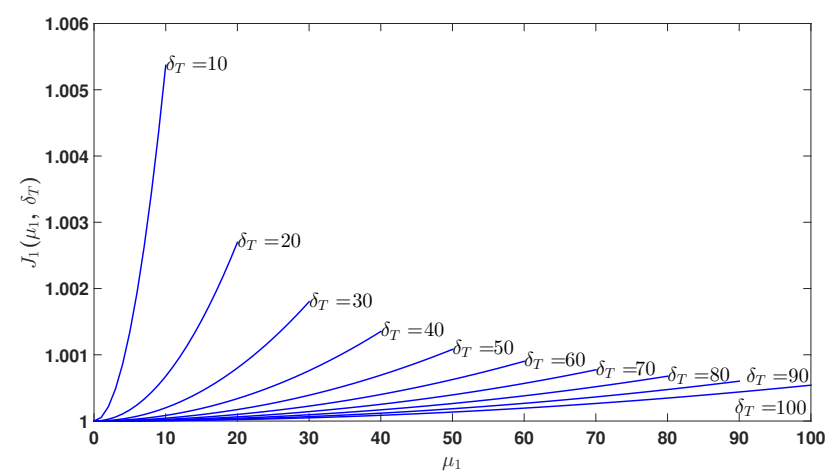

Fig. 8: Analysis of $J_{1}\left(\mu_{1}, \delta_{T}\right)$ for the graph in Fig.5

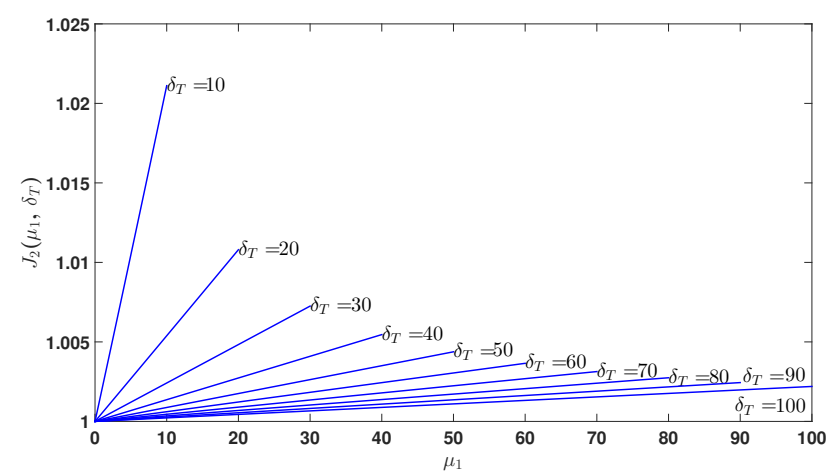

Fig. 9: Analysis of $J_{2}\left(\mu_{1}, \delta_{T}\right)$ for the graph in Fig. 5

estimate, $\gamma_{L P}$, and the estimate obtained via Lyapunov's direct method, $\gamma_{M}$. We reconsider the single-integrator agent dynamics with the interaction graph as in Fig. 5. 
For the sake of a making a coherent comparison with the $B$-estimate -see Remark 2, we normalize the upper-bound on $M(t)$ with respect to $p$, that is, we set $\mu_{2}:=\delta_{T}$. In Fig. 8 and Fig. 9 we depict the plot of $J_{1}\left(\mu_{1}, \delta_{T}\right):=e^{2\left(T \gamma_{M}-T \gamma_{B}\right)}$ and $J_{2}\left(\mu_{1}, \delta_{T}\right):=e^{2\left(T \gamma_{L P}-T \gamma_{B}\right)}$, respectively, versus $\mu_{1}$ and different values of $\delta_{T}$. Since, $\delta_{T} \geq \mu_{1}$, each curve extends only up to $\mu_{1}=\delta_{T}$. From the above simulations it may be conclude that, both, the LP-estimate, $\gamma_{L P}$, and that generated via the Lyapunov function, $\gamma_{M}$, give a tighter bound than the $B$-estimate. Note, also, that the A-estimate and the LP-estimate are equivalent for $p=1$ and $\mu_{2}=\delta_{T}$-see Remark 1.

\section{CONCLUding REMARKS}

In this work, we estimate the convergence rate for a class of consensus algorithms with persistently excited, undirected interconnection networks. The linear update laws are analyzed by transforming the node agreement problem to edge agreement by a suitable coordinate transformation. The resulting exponential stability problem for both single and double integrator agents are analyzed using different approaches based on Lyapunov theory. The aforementioned techniques allow computation of explicit bounds on convergence rates to consensus for agents communicating over an undirected, time-varying graph network. Numerical case studies compare the various estimates of convergence rate with each other. It is concluded that the A and LP estimates and provide the tightest bound on the convergence rate as compared with the other estimates proposed in this work.

\section{REFERENCES}

[1] B. D. O. Anderson. Exponential stability of linear equations arising in adaptive identification. IEEE Transactions on Automatic Control,, 22(1):83-88, Feb 1977.

[2] S. Andersson and S. P. Krishnaprasad. Degenerate gradient flows: a comparison study of convergence rate estimates. In Proc. 41st. IEEE Conf. Decision. Contr., volume 4, pages 4712-4717, 2002.

[3] K. J. Åstrom and Bohn. Numerical identification of linear dynamic systems from normal operating records. In P. H. Hammond, editor, Proc. of the 2nd IFAC Symp. on Theory of Self-adaptive Control Systems, pages 96-111, Nat. Phys. Lab., Teddington, England, 1965.

[4] V. D. Blondel, J. M. Hendrickx, A. Olshevsky, and J. N. Tsitsiklis. Convergence in multiagent coordination, consensus, and flocking. In Proceedings of the 44th IEEE Conference on Decision and Control, pages 2996-3000, Dec 2005.

[5] R. Brockett. The rate of descent for degenerate gradient flows. In Proc. Math. Theory of Networks and Systems, 2000.

[6] M. Cao, A. Stephen Morse, and B. D. O. Anderson. Reaching a consensus in a dynamically changing environment: A graphical approach. SIAM J. on Contr. and Opt., 47(2):575-600, 2008. 
[7] N. R. Chowdhury and S. Srikant. Persistence based analysis of consensus protocols for dynamic graph networks. In Proc. 13th. IEEE European. Contr. Conf., pages 886-891, 2014.

[8] N. R. Chowdhury and S. Sukumar. A comparative study of persistence based convergence rate estimates to consensus. IFAC-PapersOnLine, 48(11):534 - 539, 2015. 1st. IFAC Conf. Modelling, Identification and Control of Nonlinear Systems MICNON.

[9] D. Goldin and J. Raisch. Consensus for agents with double integrator dynamics in heterogeneous networks. AJOC, 16(1):30-39, 2014.

[10] J. Hendrickx and J. N. Tsitsiklis. Convergence of type-symmetric and cut-balanced consensus seeking systems. IEEE Trans. on Automat. Contr., 58(1):214-218, 2013.

[11] A. Loría and E. Panteley. Uniform exponential stability of linear time-varying systems:revisited. Syst. \& Contr. Letters, 47(1):13-24, 2002.

[12] S. Martin and A. Girard. Continuous-time consensus under persistent connectivity and slow divergence of reciprocal interaction weights. SIAM J. on Contr. and Opt., 51(3):2568-2584, 2013.

[13] M. Mesbahi and M. Egerstedt. Graph theoretic methods in multiagent networks. Princeton University Press, 2010.

[14] L. Moreau. Stability of multiagent systems with time-dependent communication links. IEEE Transactions on Automatic Control, 50(2):169-182, Feb 2005

[15] K. S. Narendra and A. M. Annaswamy. Stable adaptive systems. Prentice-Hall, Inc., New Jersey, 1989.

[16] A. Olshevsky and J. N. Tsitsiklis. Degree fluctuations and the convergence time of consensus algorithms. IEEE Transactions on Automatic Control, 58(10):2626-2631, Oct 2013.

[17] G. Shi and K. H. Johansson. The role of persistent graphs in the agreement seeking of social networks. IEEE. J. Selec. Areas. Coтmu., 31(9):595-606, September 2013. 